\title{
Prestress loss of prestressing tendons with asymmetrical layout parabolas
}

\author{
Shanwen Zhang ${ }^{1, a}$, Xiuxin Wang ${ }^{2, b}$ * \\ ${ }^{1,2}$ School of civil engineering, Southeast University, Nanjing 210096, China \\ axiucaiseu@163.con, bwangdisk@163.com,
}

Keywords: prestressed concrete structure; prestressing tendons; loss of prestress; stress balance point; asymmetrical layout

Abstract. According to the equilibrium condition of geometry relationship, we derived the formulas that can be used to calculate the loss of prestress of prestressing tendons with the double-end tensioning and the asymmetrical arrangement parabolic curves. Through an example of the bridge engineering, we described in detail the complete procedure of the proposed formula method, and it is shown that the proposed method is simple and practical. Comparing with the Midas software method would prove to be accurate. This work could be used to calculate the losses of prestress for 2-dimensional parabolic prestressing tendons.

\section{Introduction}

Post-tensioned prestressed members have been widely applied in the roads, the bridges, the buildings and the municipal engineering. For the prestressing tendons of parabolic curves, losses of prestress due to friction have two ways in tensioning stage: the one is the effect of the bent duct, the other is the effect of the local deviation of ducts. The effective prestressing force was very importance factor in order to ensure the construction quality of the tendons. Thus, it is vital to make more exact calculation of prestress losses. China and America bridge specifications ${ }^{[1-3]}$ gave the description of calculating prestress losses, but not specific formulas. Here, basing the equilibrium condition of geometry relationship, we would derive the basic formulas of prestress loss and give the specific formulas to calculate the parabolic bending angle and curve length. The stress balance point would be presented when the prestressing tendons adopted the double-end tensioning and asymmetrical layout. Finally, we would give an example of bridge engineering.

\section{Stress balance point}

When the prestressing tendons were the single-end tensioning or double-end tensioning and symmetrical layout, we may calculate pretress loss of every segment one by one; when the double-end tensioning and asymmetrical layout, the values are not easy to obtain. We have to calculate every segment one by one from two endpoints, respectively. Due to the friction, the prestress value of the tendons must gradually decrease a certain value in a certain point to make the system achieve balance. We called the "a certain point" as the "stress balance point" or "fixed point in theory".

It was assumed that the prestress values of two endpoints can be defined as $\sigma_{i}$ and $\sigma_{i+1}$, respectively. According to the force equilibrium condition, the following is obtained:

$$
\sigma_{i} e^{-\left(k s_{i}+u \phi_{i}\right)}=\sigma_{i+1} e^{-\left(k s_{i+1}+u \phi_{i+1}\right)}
$$

So we can get the expression:

$$
\left\{\begin{array}{c}
s_{i}+s_{i+1}=s \\
\phi_{i}+\phi_{i+1}=\phi \\
k\left(s_{i}-s_{i+1}\right)+\mu\left(\phi_{i}-\phi_{i+1}\right)=\ln \frac{\sigma_{i}}{\sigma_{i+1}}
\end{array}\right.
$$


Where, $s$ is the length of a prestressing tendon from the jacking endpoint; $\phi_{i}$ is the sum of the absolute values angular change of prestressing tendon path from the jacking endpoint ( $\mathrm{rad}) ; s_{i}, s_{i+1}$ are the length of a prestressing tendons from the jacking endpoint to the stress balance point, respectively (m); $\phi_{i}, \phi_{i+1}$ are the sum of the absolute values angular change of prestressing tendon path from jacking end, or from the jacking endpoint to the stress balance point, respectively ( $\mathrm{rad}$ ).

\section{Formulas derivation}

There is 2-d parabolic curve $\left(y=a x^{2}\right)$, the stress balance point is the point $\mathrm{O}$, as shown in figure 1 .

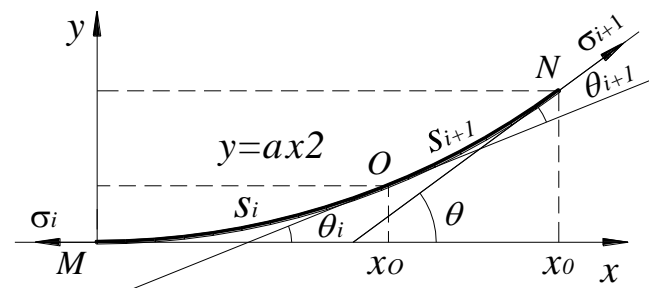

Fig. 1 The schematic diagram of parabolic curve and stress balance point $\mathrm{O}$

According to the Frenet formulas of differential geometry theory, we can obtain the 2-d general expressions for the curvature, bending angle $\phi$ and curve length $s$ as:

$$
\begin{aligned}
& k(s)=\frac{x^{\prime} y^{\prime \prime}-y^{\prime} x^{\prime \prime}}{\left(x^{\prime 2}+y^{\prime 2}\right)^{3 / 2}} \\
& s=\int_{0}^{x_{0}} \sqrt{x^{\prime 2}+y^{\prime 2}} d x \\
& \phi=\int_{0}^{x_{0}} \frac{x^{\prime} y^{\prime \prime}-y^{\prime} x^{\prime \prime}}{x^{\prime 2}+y^{\prime 2}} d x
\end{aligned}
$$

On the basis of the Eq. (4), the expression of curve length may be taken as:

$$
s=\int_{0}^{x_{0}} \sqrt{1+\left(2 a_{1} x\right)^{2}} d x=\frac{x_{0}}{2} \sqrt{4 a_{1}^{2} x_{0}^{2}+1}+\frac{1}{4 a_{1}} \ln \left(2 a_{1} x_{0}+\sqrt{4 a_{1}^{2} x_{0}^{2}+1}\right)
$$

On the basis of the Eq.(5), the expression of bending angle may be taken as:

$$
\phi=\int_{0}^{x_{0}} \frac{2 a_{1}}{1+\left(2 a_{1} x_{0}\right)^{2}} d x=\arctan \left(2 a_{1} x_{0}\right)
$$

Reference the figure 7 , considering the tendons layout is general flat, the curvature radius of the parabola may be replaced by the value of the vertex.

Substituting $x=0$ into the Eq.(3):

$$
k(s)=\frac{y^{\prime \prime}}{\left(1+y^{\prime 2}\right)^{3 / 2}}=2 a
$$

The curvature radius of the parabola is

$$
r=\frac{1}{k(s)}=\frac{1}{2 a}
$$

The curve length

$$
s=\frac{1}{2 a} \cdot \theta
$$

By substituting the Eq. (9) and Eq. (10) into the Eq.(2), the following is obtained:

$$
\theta_{O}=\frac{a}{k+2 \mu a}\left(\frac{k}{2 a}+\mu \theta+\ln \frac{\sigma_{i}}{\sigma_{i+1}}\right)
$$




$$
x_{O}=\frac{1}{2 a} \cdot \tan \theta_{O}
$$

\section{Example and Discussion}

An example is a prestressed concrete bridge of continuous curved box-girder, which is $12 \mathrm{~m}$ wide. The span distributions are $28 \mathrm{~m}$ and $24 \mathrm{~m}$. The drawing of the curved bridge and prestressing tendons $\mathrm{N} 1$ referred to the figure 2. The projection of the tendons $\mathrm{N} 1$ is composed of multiple lines and parabolic curves in the horizontal plane. The curve segments have BC, DE, EG, GH and IJ, the line segments have AB, CD, HI, and JK, as shown in the figure 3. Every parabolic segment has the same curvature, the parabolic equation expressions may be written as: $\mathrm{Y}=0.08 \mathrm{X}^{2}, \mathrm{y}=-0.08 \mathrm{x}^{2}$, and their own vertex are defined as the coordinate origin, respectively. The tendons grade is $10 \times 7-\phi^{\mathrm{s}} 15.24-1860$, the tensile stresses is $1395(\mathrm{MPa})$, the coefficient of $k$ is 0.0015 , and $\mu$ is 0.20 .
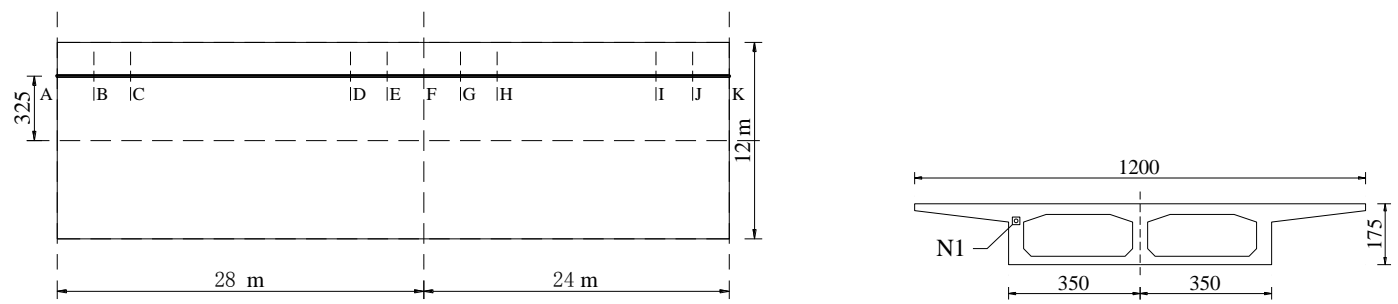

Fig. 2 The schematic diagram of the bridge layout and prestressing tendons unit: $\mathrm{cm}$

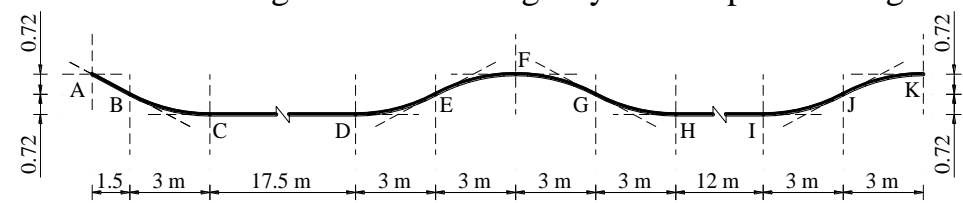

Fig.3 The dimensioned drawing of the tendons on the vertical plane of projection

The formula method. We need to divide into ten segments according to the characteristics of the tendons. Then, we have to calculate every segment one by one and find the location of the stress balance point. By using the Eq.(6), and Eq. (7), we can calculate the prestress losses from the point A to K and point $\mathrm{K}$ to A, respectively. The obtained data of nodes would draw two monotonic decreasing curves, as shown in figure 4 .
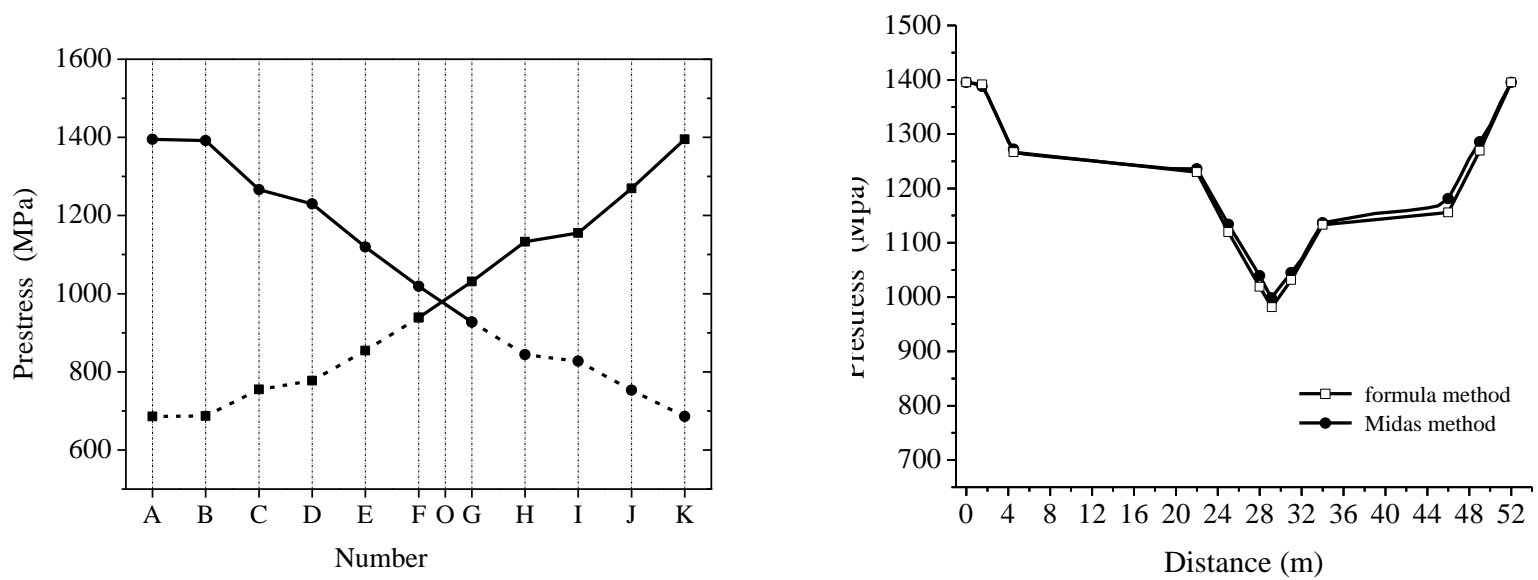

Fig. 4 One-way prestress loss curves from endpoints Aand K Fig. 5 Pretress loss curves of prsetressing tendons of two methods

The balance point $O$ is between the points $F$ and G, by solving the Eq. (2), Eq. (10), Eq. (11), we may obtain the values for $\phi_{i}$ and $s_{i}$.

The Midas method and discussion. The finite element method takes the Midas/civil software, we define that the anchorage deformation is zero, the control stresses of the endpoints A and K are 1395 (MPa), the endpoints $\mathrm{A}$ and $\mathrm{K}$ are uniform loaded at the same time. In order to comparison and analysis, two loss curves 
of two methods were drawed in the same digram, as shown in the figure 5. For the sake of analyzing the diference in point $\mathrm{O}$, the obtained datas were listed in the table 1 .

Tab. 1 Pretress values and location of the point $\mathrm{O}$ of two methods

\begin{tabular}{cccc}
\hline Methods & $\begin{array}{c}\text { Segment AO } \\
\text { Arc length } / \mathrm{m}\end{array}$ & $\begin{array}{c}\text { Point O stress } \\
\text { /MPa }\end{array}$ & $\begin{array}{c}\text { Prestress loss } \\
\text { /MPa }\end{array}$ \\
\hline Midas method & 29.103 & 998.44 & 396.56 \\
Formula method & 29.167 & 981.15 & 413.85 \\
D-value & 0.064 & 17.29 & 17.29 \\
\hline
\end{tabular}

From the above figure 5, we can find that the prestress loss curve of the formula method is in good agreement with the Midas method, the values have little difference between the two calculation methods. Refer to the table 1, the numbers show that there is 17.29 (MPa) difference for the stress value in the fixed point $\mathrm{O}$ between two methods, it is only 1.74 per cent of the Midas method; the length deviation of the segment $\mathrm{AO}$ is $0.064(\mathrm{~m})$ difference, it is only 0.22 per cent of that.

These data indicate that the difference is very little between the two calculation methods. The little error possibly source from the accumulation of rounding error and (or) density of element division, et al. Therefore, by comparing the two methods, the Formula method is proved to be accurate.

\section{Conclusions}

In this paper, we have analyzed the two-dimension parabolic curves which were normally used in the prestressed concrete structure. Based on the equilibrium condition of geometry relationship, the stress balance point was presented when the prestressing tendons in tensioning stage were the double-end tensioning and asymmetrical layout, the specific equations were derived to solve the corresponding angle and curve length for the two-dimensional parabolic curves.

Through an example of the bridge engineering, we described in detail the complete procedure of the proposed formula method, and it is shown that the proposed method is simple and practical. By compared with the Midas software method, the accuracy of formula method can be proved. This work could be used to calculate the losses of prestress for 2-dimensional parabolic curves prestressing tendons in prestressed concrete structure.

\section{References}

[1] JTG/T F50-2011, Technical Specification For Construction of Highway Bridge and Culverts, China Communications Press, Beijing, 2011. (in Chinese)

[2] JTG D62-2004, Code for design of highway reinforced concrete and prestressed concrete bridge and culverts, China Communications Press, Beijing, 2004. (in Chinese)

[3] AASHTO Committee, AASHTO LRFD Bridge Design Specification, The American Association of State Highway and Transportation Officials, Washington. DC, 2007.

[4] T. Y. Lin, Ned H. Burns, Design of Prestressed Concrete Structures (third edition), John Wiley \& Sons, New York, 1981.

[5] Song Yupu, Che Yi, MaDeyou, Zhao Guofan. Stud on losses of prestress due to friction for curved prestressing tendon, China civil engineering journal, 2002, vol.35, No.6:105-108.(in Chinese)

[6] Zhang Zhihua, Computation of stretching extension of post-tensioned prestressed reinforcing bar, Construction technology, 2011, (40):49-51. (in Chinese) 